\title{
A History and Overview of Video Game Addiction
}

Mark D. Griffiths and Halley M. Pontes

\begin{abstract}
The past decade has witnessed a significant increase in the number of empirical studies examining various aspects of problematic video game play, video game addiction, and, more recently, gaming disorder. This chapter begins with a brief past history of how research into video game addiction has developed during the past four decades in the 1980s (arcade video game addiction), 1990s (home console video game addiction), and 2000 s and beyond (online video game addiction). The chapter also overviews the features of gaming addiction, its prevalence rates, demographics and gaming addiction, negative consequences of excessive video game use, Internet gaming disorder and the DSM-5, and treatment of gaming addiction. Based on the published evidence, particularly from studies conducted in the past decade, it appears that, in extreme cases, excessive gaming can have potentially damaging effects on individuals who appear to display compulsive and/or addictive behavior similar to other more traditional addictions. However, the field has been hindered by the use of inconsistent and nonstandardized criteria to assess and identify problematic and/or addictive video game use.
\end{abstract}

Keywords: excessive gaming, video game addiction, gaming addiction, gaming disorder, Internet gaming disorder, problematic gaming

According to the Entertainment Software Association (ESA, 2014), 59\% of the entire American population plays video games, with an average of two gamers in each game-playing household. Additionally, among US households $68 \%$ play video games on consoles, $53 \%$ play on smartphones, and $41 \%$ play on wireless devices (ESA, 2014). During 2012, playing video games via smartphones and wireless devices increased by $22 \%$ and $37 \%$, respectively. The average video game player is 31 years old - and about $52 \%$ of these video game players are male and $48 \%$ are female (ESA, 2014).

Given the pervasiveness of gaming across several countries and different segments of the population, the study of its associated effects on general human behavior, physical and mental health has become an important topic for dedicated research agendas from various scientific domains in addition to psychology and psychiatry (e.g., Blocher, 2015; Dreier, Wölfling, \& Müller, 2013; Johnston, Boyle, MacArthur, \& Manion, 2013). Taken together, findings in this developing field suggest both favorable and unfavorable effects of gaming, outcomes that could inform decisions made by healthcare professionals, parents, policymakers (Przybylski, 2014) and other stakeholders such as researchers and the video game industry (Yousafzai, Hussain, \& Griffiths, 2013).

One topic that has become of increasing research interest is gaming addiction and Internet Gaming Disorder (IGD). The past decade has witnessed a significant increase in the number of empirical studies examining various aspects of problematic video gameplay and video game addiction (Pontes \& Griffiths, 2014). This chapter begins with a brief past history of how research into video game 
addiction has changed over the past three decades (i.e., the 1980s, 1990s, and 2000s). The chapter addresses concerns related to video game addiction and how it made its way into the latest (fifth) edition of the DSM (DSM-5; American Psychiatric Association, 2013). The chapter also briefly examines features of video game addiction and examines the contemporary research literature by analyzing the prevalence of video game addiction, factors associated with video game addiction, and the treatment of video game addiction.

\section{Gaming Addiction in the 1980s}

Following the release of the first commercial video games in the early 1970s, it took until the 1980s for the first reports of video game addiction to appear in the psychological and psychiatric literature. In the early 1980s, Ross, Finestone, and Lavin reported three cases of "Space Invaders obsession" and Nilles (1982) described a similar phenomenon but called it "computer catatonia." Arguably the first reference to "video game addiction" was by Soper and Miller (1983) who, based on their observations as school counselors, claimed the disorder was like any other behavioral addiction and consisted of a compulsive behavioral involvement, a lack of interest in other activities, association mainly with other addicts, and physical and mental symptoms when attempting to stop the behavior (e.g., the shakes). Some credence was given to these claims that video game addiction existed following papers on the seemingly successful treatment of video game addiction using cognitive behavioral therapy (CBT) (Keepers, 1990; Kuczmierczyk, Walley, \& Calhoun, 1987). However, all of these studies were somewhat observational, anecdotal, and/or case studies, primarily based on teenage males and all based on a particular type of video game in a particular medium (i.e., "pay-to-play" arcade video games).

Shotton (1989) carried out the first empirical study specifically on gaming addiction on a relatively small sample of 127 people (almost all teenage or young adult males) who described themselves as "hooked" on home video games for at least 5 years. Shotton's conceptualization of gaming addiction was more positive than negative, and she reported that her "addicts" were on the whole highly intelligent, motivated, and achieving people who were often misunderstood by others in society. Despite this, the main problem with the study was that no standardized measure of addiction was actually used. The only criterion for being "addicted" was the individuals own admission that they were "hooked" on computer games. Despite this major shortcoming, recent research by Widyanto, Griffiths, and Brunsden (2011) reported that a person's positive self-diagnosis to the Internet was correlated highly with more standardized measures of Internet addiction.

\section{Gaming Addiction in the 1990s}

The 1990s saw a small but significant increase of research into video game addiction, with almost all of these studies being carried out by surveying children in school settings in the United Kingdom (e.g., Brown \& Robertson, 1993; Fisher, 1994; Griffiths, 1997; Griffiths \& Hunt, 1995, 1998; Parsons, 1995; Phillips, Rolls, Rouse, \& Griffiths, 1995). In contrast to studies from the early 1980s, these studies mainly examined non-arcade video game playing (i.e., home console games, handheld games, PC gaming). However, all of these studies were self-report surveys, relatively small in scale, and all of them assessed video game addiction using adapted versions of the Diagnostic and Statistical Manual of Mental Disorders (DSM), such as the DSM-III or the DSM-IV criteria for pathological gambling (American Psychiatric Association, 1987, 1994). Based on further analysis of the adapted DSM criteria used, these studies were later criticized as being more likely to be assessing video game preoccupation rather than video game addiction (Charlton, 2002).

\section{Gaming Addiction in the 2000s and Beyond}

The 2000s saw a substantial growth in the number of studies on video game addiction, particularly as gaming expanded into the new online medium where games could be played as part of a gaming community (i.e., massively multiplayer online role playing games [MMORPGs] such as World of Warcraft and Everquest). Approximately 60 studies were published on gaming addiction between 2000 and 2010 (Kuss \& Griffiths, 2012) and a vast majority of these studies examined MMORPG addiction and were not limited to only studying adolescent males. Furthermore, many of these studies collected their data online, and a significant number of studies examined various aspects of video game addiction using non-self-report methodologies. These include studies using polysomnographic measures and visual and verbal memory tests (Dworak, Schierl, Bruns, \& Struder, 2007); medical examinations including the patient's history, and physical, radiologic, intraoperative, and pathologic findings (Cultrara \& Har-El, 2002); functional magnetic resonance imaging (fMRI; Han, Hwang, \& 
Renshaw, 2010; Hoeft, Watson, Kesler, Bettinger, \& Reiss, 2008; Ko et al., 2009); electroencephalography (Thalemann, Wölfling, \& Grüsser, 2007); and genotyping (Han et al., 2007). Given the methodological shortcomings of the studies published prior to 2000 and the fact that gaming has evolved substantially over the past decade, the remainder of this chapter will mainly focus on studies published in the past decade or so (i.e., post-2000 studies) with the exception of those concerning the health and medical consequences of excessive video game play.

\section{Features of Gaming Addiction}

There are a multitude of psychological perspectives on addiction, which has led to addiction being defined in many different ways. However, most models of addictive behavior refer to a persistent and uncontrollable urge to consume a substance or engage in an activity that results in significant personal harm and interpersonal conflict for the user (King, Delfabbro, $\&$ Griffiths, 2013). Thus, gaming addiction is often said to be present when individuals have completely lost control over gaming and the excessive playing behavior has had a detrimental effect on all aspects of the individuals life, compromising job and/or educational activities, interpersonal relationships, hobbies, general health, and psychological well-being (King, Delfabbro, \& Griffiths, 2013). These two criteria (impaired control and harmful consequences) are regarded as fundamentally important criteria for addiction. An alternative model of addictive behavior has proposed six features or components of gaming addiction (Griffiths, 2005). To indicate addiction, it is thought that these criteria must be sustained for at least 3-6 months. Otherwise, they may simply indicate a temporary absorption in video games. These criteria include:

- Salience. This occurs when gaming becomes the most important activity in a person's life, dominating thoughts (preoccupation and cognitive distortions), emotions (cravings), and behavior (deterioration of normal behaviors). An addicted gamer is obsessed with all aspects of video games and, when not playing, will be anticipating or planning the next playing session.

- Mood modification. This refers to changes in a person's mood state that occur as a result of gaming. Mood change may involve a subjective feeling of euphoria as well as an increase in physiological arousal (increased heart rate, muscle tension, or shaky hands) or, alternatively, a tranquilizing feeling of calm or a numbing sensation.
- Tolerance. This refers to the process whereby increasing amounts of gaming are required to achieve the former mood-modifying effects. This means that players gradually increase the amount of time they spend engaged in gaming. It could be argued that addicted gamers build up their tolerance to the point that they will end a playing session only when they have become mentally or physically exhausted.

- Withdrawal. These are the aversive mood states and/or physical effects that occur when gaming is suddenly discontinued or reduced. Psychological withdrawal symptoms include feelings of frustration, irritability, and flattened affect. Withdrawal motivates the individual to play video games on a regular basis and to minimize periods of absence from a video game in order to alleviate these unpleasant feeling states.

- Relapse. This refers to the tendency for the player to make repeated reversions to earlier patterns of gaming and for even the most extreme patterns typical of the height of excessive gaming to be quickly restored after periods of abstinence or moderation. Relapse usually indicates that the individual has lost personal agency over the behavior.

- Conflict (harm). This refers to the negative consequences of excessive gaming. Harm includes conflicts between the addicted video game player and other people (family members and friends), other activities (job, school, social life, hobbies and interests), and from within the addict him/herself (psychological distress).

Charlton (2002) suggests that three of these features may not be reliable indicators of video game addiction. His research suggests that cognitive salience (preoccupation), euphoria (mood modification), and tolerance also indicate high engagement, or a type of healthy obsession, with gaming. Therefore, studies may overestimate the prevalence of problem video game play if high engagement with gaming is not properly distinguished from gaming addiction. Given these issues of reliability, many addiction specialists maintain that impaired control and harmful effects are the most appropriate criteria for identifying gaming addiction.

\section{Prevalence of Problematic Video Game Use and Gaming Addiction}

At present, it is quite difficult to estimate the prevalence of problematic gaming due to the lack of a clear definition, the application of measures without proper psychometric characteristics, and studies 
using different samples and different research methodologies. Large-sample studies generally report prevalence values of lower than $10 \%$. A study conducted in the United States on a national representative sample of teenagers (Gentile, 2009), as well as on a large sample of Singaporean children (Gentile et al., 2011) both reported a problematic game use of approximately $9 \%$. Results of another representative study in Germany showed that 3\% of the male and $0.3 \%$ of the female students studied were diagnosed as dependent on video games, while another $4.7 \%$ of male and $0.5 \%$ of female students were at risk of becoming dependent (Rehbein et al., 2010). On a large Hungarian online gamer sample $3.4 \%$ of gamers belonged to the high-risk group of problematic gaming and another $15.2 \%$ to the medium-risk group (Demetrovics et al., 2012). A proportion of $4.6 \%$ of Hungarian adolescents (approximately 16 years old) belonging to a national sample were classified as high-risk users (Pápay et al., 2013) (see Table 2.1).

\section{Demographics and Gaming Addiction}

According to an online survey examining all types of online gamers (Nagygyörgy et al., 2013) $(N=4,374)$, the mean age was 21 years, and participants were mostly male (91\%) and single (66\%). Their average weekly game time varied between less than 7 hours (10\%) and more than 42 hours (also 10\%) with most of the gamers playing 15-27 hours weekly (35\%). Furthermore, 16\% of all gamers were playing either professionally (i.e., they make a living off of sponsorships and money won from tournaments) or competitively (i.e., they participate in competitions and earn money if they win). The majority of the sample $(79 \%)$ had a clear gaming preference: namely, they played one single game type most of the time.

Data regarding the three main game types give a more nuanced view. The proportion of female gamers is the lowest in the case of massively multiplayer online first-person shooter (MMOFPS) games (1-2\%) (Jansz \& Tanis, 2007; Nagygyörgy et al., 2013) and the highest between MMORPG users (15-30\%) (Cole \& Griffiths, 2007; Nagygyörgy et al., 2013; Yee, 2006a). MMOFPS users are the youngest (18-19.8 years) (Jansz \& Tanis, 2007; Nagygyörgy, et al., 2013), while both massively multiplayer online real-time strategy (MMORTS) (22 years) (Nagygyörgy et al., 2013) and MMORPG players (21-27 years) (Nagygyörgy et al., 2013; Yee, 2006a) are significantly older. Among the three main groups, MMORPG gamers spend the most time playing (Nagygyörgy et al., 2013). Since MMORPGs are the most researched games (most likely because they allow players to interact to form friendships, create communities, and work together to accomplish a variety of goals [Barnett \& Coulson, 2010]), there is additional information regarding such players that is still unknown in the case of other game types. For instance, half of MMORPG players work full time, 22.2\% are students, and $14.8 \%$ are homemakers $(89.9 \%$ of whom were female). Furthermore, 36\% of the gamers are married and $22 \%$ of them have children (Yee, 2006a, 2006b). Overall, the demographic composition of MMORPG users is quite varied and probably more diverse than the composition of MMORTS and MMOFPS users (although this needs to be empirically established).

From a substantive perspective, there are some generalizations that can be made with regard to the demographic characteristics of gamers and problem gamers. The literature to date suggests that adolescent males and young male adults appear to be at greater risk of experiencing problematic video game play. However, the course and severity of these problems is not well known (King, Delfabbro, \& Griffiths, 2012) and the finding that this group is more at risk may be a consequence of sampling bias and the fact that this group plays video games more frequently than do other sociodemographic groups. It has also been suggested that university students may be vulnerable to developing problematic video gaming. Reasons for this include their flexible tuition and study hours, ready access to high-speed broadband on a 24/7 basis, and multiple stressors associated with adjusting to new social obligations and/or living out-of-home for the first time (King, Delfabbro et al., 2012; Young, 1998a).

\section{Negative Consequences of Excessive Video Game Use}

Irrespective of whether problematic video game play can be classed as an addiction, there is now a relatively large number of studies all indicating that excessive video game play can lead to a wide variety of negative psychosocial consequences for a minority of affected individuals. These include sacrificing work, education, hobbies, socializing, time with partner/family, and sleep (Batthyány, Müller, Benker, \& Wölfling, 2009; Griffiths, Davies, \& Chappell, 2004; King \& Delfabbro, 2009; Liu \& Peng, 2009; Peng \& Liu, 2010; Peters \& Malesky, 2008; Rehbein et al., 2010; Yee 2006a, 2006b), increased stress (Batthyány et al., 2009), an absence of real-life relationships (Allison, von Wahlde, Shockley, \& Gabbard, 2006), lower 
Table 2.1 Prevalence of problematic online gaming involving large samples

\begin{tabular}{|c|c|c|c|c|c|c|}
\hline $\begin{array}{l}\text { Author(s) } \\
\text { (reference) }\end{array}$ & Location & $\begin{array}{l}\text { Research subjects } \\
\text { (mean age }(\mathrm{M}) \text {, } \\
\text { standard deviation }(\mathrm{SD}) \text { ) }\end{array}$ & Method & Measure & $\begin{array}{l}\text { Criteria of } \\
\text { problematic use }\end{array}$ & $\begin{array}{l}\text { Life-prevalence } \\
\text { value }\end{array}$ \\
\hline Yee (2006) & USA, Canada & $\begin{array}{l}\text { 3,166 persons, MMORPG } \\
\text { gamers }\end{array}$ & Online survey & $\begin{array}{l}\text { Direct question ("Do you } \\
\text { consider yourself addicted to } \\
\text { MMORPGs?" yes/no question) }\end{array}$ & $\begin{array}{l}\text { Yes to the direct } \\
\text { question }\end{array}$ & $50 \%$ \\
\hline $\begin{array}{l}\text { Grüsser, } \\
\text { Thalemann, } \\
\text { and Griffiths } \\
\text { (2007) }\end{array}$ & Germany & $\begin{array}{l}7,069 \text { gamers (M: } 21,1 \text { years, } \\
\text { SD: } 6,4)\end{array}$ & Online survey & $\begin{array}{l}6 \text { criteria of key symptoms of } \\
\text { a dependence syndrome as } \\
\text { outlined in WHO's ICD-10 }\end{array}$ & $\begin{array}{l}3 \text { or more criteria } \\
\text { fulfilled }\end{array}$ & $11,9 \%$ \\
\hline Gentile (2009) & USA & $\begin{array}{l}1,178 \text { persons (adolescents } \\
\text { aged } 8-18 \text { years) }\end{array}$ & $\begin{array}{l}\text { Nationally } \\
\text { representative } \\
\text { online survey }\end{array}$ & Pathological Video-Game Use & 6 or more yes answers & $8,5 \%$ \\
\hline $\begin{array}{l}\text { Porter et al. } \\
(2010)\end{array}$ & $\begin{array}{l}\text { USA, Canada, } \\
\text { Europe, Australia, } \\
\text { New Zeeland, Asia, } \\
\text { Central and South } \\
\text { America }\end{array}$ & $\begin{array}{l}1,945 \text { persons, gamers older } \\
\text { than } 14 \text { years }\end{array}$ & Online survey & $\begin{array}{l}\text { Video Game Use } \\
\text { Questionnaire, (VGUQ), } 10 \\
\text { criteria: } 3 \text { (preoccupation), } 7 \\
\text { (adverse consequences), } \\
\text { yes/no answers }\end{array}$ & $\begin{array}{l}2 \text { or more criteria } \\
\text { (preoccupation) }+3 \text { or } \\
\text { more criteria (adverse } \\
\text { consequences) }\end{array}$ & $8 \%$ \\
\hline $\begin{array}{l}\text { Van Rooij et al. } \\
\text { (2011) }\end{array}$ & Netherlands & $\begin{array}{l}4,559 \text { persons (M: } 14.4 \text { years, } \\
\text { SD: } 1.2)(\mathrm{T} 1) \text { and } 3740 \\
\text { persons (M: } 14.3 \text { years } \\
\text { SD: } 1.0 \text { ) (T2) }\end{array}$ & $\begin{array}{l}\text { Paper-and-pencil } \\
\text { survey }\end{array}$ & $\begin{array}{l}\text { Compulsive Internet Use Scale } \\
\text { (CIUS) + weekly hours online } \\
\text { gaming }\end{array}$ & Latent profile analysis & $\begin{array}{l}1,6 \%(\mathrm{~T} 1), 1,5 \% \\
(\mathrm{~T} 2)-1,5 \%\end{array}$ \\
\hline $\begin{array}{l}\text { Gentile et al. } \\
(2011)\end{array}$ & Singapore & $\begin{array}{l}3,034 \text { persons elementary } \\
\text { and secondary school } \\
\text { students }\end{array}$ & $\begin{array}{l}2 \text { years longitudinal } \\
\text { study paper-and- } \\
\text { pencil survey }\end{array}$ & Pathological Video-Game Use & 5 or more yes answer & $7,6 \%-9,9 \%$ \\
\hline $\begin{array}{l}\text { Lemmens, } \\
\text { Valkenburg, } \\
\text { and Peters } \\
\text { (2011) }\end{array}$ & Netherlands & $\begin{array}{l}543 \text { adolescent gamers } \\
\text { (M: } 13.9 \text { years, SD: } 1.4)\end{array}$ & $\begin{array}{l}\text { Longitudinal } \\
\text { paper-and-pencil } \\
\text { survey }\end{array}$ & Game Addiction Scale (GAS) & Score of 3 or higher & $6 \%(\mathrm{~T} 1), 4 \%(\mathrm{~T} 2)$ \\
\hline $\begin{array}{l}\text { Rehbein et al. } \\
(2010)\end{array}$ & Germany & $\begin{array}{l}15,168 \text { ninth graders }(15.3 \\
\text { years, SD: } 0.69)\end{array}$ & $\begin{array}{l}\text { Nationally } \\
\text { representative } \\
\text { survey }\end{array}$ & $\begin{array}{l}\text { Video Game Dependency Scale } \\
\text { (KFN-CSAS-II) }\end{array}$ & $\begin{array}{l}\text { Score higher than } 42 \\
\text { dependent players; } \\
\text { score between } 35 \text { and } \\
\text { 41: players at risk }\end{array}$ & $\begin{array}{l}\text { dependent: boys } 3 \% \text {, } \\
\text { girls } 0,3 \% \text {; at risk: } \\
\text { boys } 4,7 \% \text {, girls } 0,5 \%\end{array}$ \\
\hline
\end{tabular}




\begin{tabular}{|c|c|c|c|c|c|c|}
\hline $\begin{array}{l}\text { Thomas and } \\
\text { Martin (2010) }\end{array}$ & Australia & $\begin{array}{l}2,031 \text { persons (705 university } \\
\text { students, } 1,326 \text { secondary } \\
\text { and college students) }\end{array}$ & $\begin{array}{l}\text { Paper-and-pencil } \\
\text { survey }\end{array}$ & $\begin{array}{l}\text { Adaptation of YDQ (Young's } \\
\text { Diagnostic Questionnaire) to } \\
\text { computer games }\end{array}$ & Score of 5 or higher & $5 \%$ \\
\hline $\begin{array}{l}\text { Jeong and Kim } \\
\text { (2011) }\end{array}$ & South Korea & $\begin{array}{l}600 \text { persons } \\
\text { (12-18 years) }\end{array}$ & $\begin{array}{l}\text { Nationally } \\
\text { representative } \\
\text { paper-and-pencil } \\
\text { survey }\end{array}$ & $\begin{array}{l}\text { Young's internet Addiction Test } \\
\text { (IAT) modified by replacing } \\
\text { the word "Internet" with } \\
\text { "gaming" }\end{array}$ & Score above 80 & $2,2 \%$ \\
\hline $\begin{array}{l}\text { Demetrovics } \\
\text { et al. (2012) }\end{array}$ & Hungary & $\begin{array}{l}\text { 3,415 persons, online gamers } \\
\text { (M: } 21 \text { years, } S D: 5,9)\end{array}$ & Online survey & $\begin{array}{l}\text { Problematic Online Gaming } \\
\text { Questionnaire (POGQ) }\end{array}$ & Latent profile analysis & $\begin{array}{l}\text { high risk problematic } \\
\text { online gamers: } 3,4 \% \text {; } \\
\text { medium risk of prob- } \\
\text { lematic use: } 15,2 \%\end{array}$ \\
\hline $\begin{array}{l}\text { Pápay et al. } \\
\text { (2013) }\end{array}$ & Hungary & $\begin{array}{l}5,045 \text { students from } \\
\text { secondary general and } \\
\text { secondary vocational schools } \\
\text { (M: } 16.4 \text { years, SD: } 0.9 \text { ) }\end{array}$ & $\begin{array}{l}\text { Nationally } \\
\text { representative } \\
\text { survey }\end{array}$ & $\begin{array}{l}\text { Problematic Online Gaming } \\
\text { Questionnaire Short Form } \\
\text { (POGQ-SF) }\end{array}$ & Latent profile analysis & $4.6 \%$ \\
\hline $\begin{array}{l}\text { Strittmatter } \\
\text { et al. (2015) }\end{array}$ & $\begin{array}{l}\text { Estonia, Germany, } \\
\text { Italy, Romania, } \\
\text { Spain, and Sweden }\end{array}$ & $\begin{array}{l}\text { 8,807 European adolescents } \\
\text { (M: } 15 \text { years, SD: } 1.3 \text { ) }\end{array}$ & $\begin{array}{l}\text { Nationally } \\
\text { representative } \\
\text { survey }\end{array}$ & $\begin{array}{l}\text { Adaptation of YDQ (Young's } \\
\text { Diagnostic Questionnaire) to } \\
\text { computer games }\end{array}$ & Score of 5 or higher & $3.1 \%$ \\
\hline $\begin{array}{l}\text { Baggio et al. } \\
(2015)\end{array}$ & Switzerland & $\begin{array}{l}5,663 \text { young adults aged } \\
\text { between } 17,14 \text {, and } 20 \text { years }\end{array}$ & $\begin{array}{l}\text { Nationally } \\
\text { representative } \\
\text { survey }\end{array}$ & GAS & $\begin{array}{l}\text { Responding } \\
\text { "sometimes" or } \\
\text { more on all } 7 \text { items }\end{array}$ & $2.3 \%$ \\
\hline $\begin{array}{l}\text { Brunborg et al. } \\
\text { (2015) }\end{array}$ & Norway & $\begin{array}{l}\text { 10,081 individuals from the } \\
\text { general population (M: } 35 \\
\text { years, SD: 13.76) }\end{array}$ & $\begin{array}{l}\text { Nationally } \\
\text { representative } \\
\text { survey }\end{array}$ & GAS & $\begin{array}{l}\text { Responding } \\
\text { "sometimes" or } \\
\text { more on all } 7 \text { items }\end{array}$ & $0.7 \%$ \\
\hline $\begin{array}{l}\text { Müller et al. } \\
\text { (2015) }\end{array}$ & $\begin{array}{l}\text { Germany, Greece, } \\
\text { Iceland, Netherlands, } \\
\text { Poland, Romania, } \\
\text { and Spain }\end{array}$ & $\begin{array}{l}\text { 12,938 European adolescents } \\
\text { aged between (M: } 15.8 \text { years, } \\
\text { SD: } 0.7 \text { ) }\end{array}$ & $\begin{array}{l}\text { Nationally } \\
\text { representative } \\
\text { survey }\end{array}$ & $\begin{array}{l}\text { Assessment of Internet } \\
\text { and Computer game } \\
\text { Addiction-Scale (AICA-S) }\end{array}$ & Score above 13.5 & $1.6 \%$ \\
\hline $\begin{array}{l}\text { Henchoz et al. } \\
\text { (2016) }\end{array}$ & Switzerland & $\begin{array}{l}5,990 \text { young adults (M: } \\
19.95 \text {, SD: } 1.2 \text { ) }\end{array}$ & $\begin{array}{l}\text { Nationally } \\
\text { representative } \\
\text { survey }\end{array}$ & GAS & $\begin{array}{l}\text { Scores of } 3 \text { or more on } \\
\text { at least any four of the } \\
\text { seven criteria }\end{array}$ & $2.2 \%$ \\
\hline
\end{tabular}


Table 2.1 Continued

\begin{tabular}{|c|c|c|c|c|c|c|}
\hline $\begin{array}{l}\text { Author(s) } \\
\text { (reference) }\end{array}$ & Location & $\begin{array}{l}\text { Research subjects } \\
\text { (mean age }(\mathrm{M}) \text {, } \\
\text { standard deviation }(\mathrm{SD}) \text { ) }\end{array}$ & Method & Measure & $\begin{array}{l}\text { Criteria of } \\
\text { problematic use }\end{array}$ & $\begin{array}{l}\text { Life-prevalence } \\
\text { value }\end{array}$ \\
\hline $\begin{array}{l}\text { Lemmens et al. } \\
(2016)\end{array}$ & Netherlands & $\begin{array}{l}2,442 \text { individuals from the } \\
\text { general population with ages } \\
\text { between } 13 \text { to } 14 \text { years }\end{array}$ & $\begin{array}{l}\text { Nationally } \\
\text { representative } \\
\text { survey }\end{array}$ & $\begin{array}{l}\text { The Internet Gaming Disorder } \\
\text { Scale (IGDS) }\end{array}$ & $\begin{array}{l}\text { Endorsing at least } 5 \\
\text { criteria }\end{array}$ & $5.8 \%$ \\
\hline $\begin{array}{l}\text { Pontes et al. } \\
(2016)\end{array}$ & Slovenia & 1,071 adolescents & $\begin{array}{l}\text { Nationally } \\
\text { representative } \\
\text { survey }\end{array}$ & $\begin{array}{l}\text { Internet Gaming Disorder } \\
\text { Scale - Short-Form } \\
\text { (IGDS9-SF) }\end{array}$ & $\begin{array}{l}\text { Endorsing at least } 5 \\
\text { criteria }\end{array}$ & $2.5 \%$ \\
\hline $\begin{array}{l}\text { Ustinavičienè } \\
\text { et al. (2016) }\end{array}$ & Lithuania & $\begin{array}{l}1,806 \text { young adolescents aged } \\
\text { between } 13 \text { to } 18 \text { years }\end{array}$ & $\begin{array}{l}\text { Nationally } \\
\text { representative } \\
\text { survey }\end{array}$ & $\begin{array}{l}\text { Adaptation of YDQ (Young's } \\
\text { Diagnostic Questionnaire) to } \\
\text { computer games }\end{array}$ & Score of 5 or higher & $9.3 \%$ \\
\hline Yu et al. (2016) & South Korea & $\begin{array}{l}\text { 2,024 young adolescents } \\
\text { aged between } 13 \text { to } 15 \text { years } \\
\text { (M: } 15.5, S D: 0.50)\end{array}$ & $\begin{array}{l}\text { Nationally } \\
\text { representative } \\
\text { survey }\end{array}$ & DSM-5 criteria for IGD & $\begin{array}{l}\text { Endorsing at least } 5 \\
\text { criteria }\end{array}$ & $5.9 \%$ \\
\hline $\begin{array}{l}\text { Carras et al. } \\
(2017)\end{array}$ & Netherlands & $\begin{array}{l}9,773 \text { adolescents } \\
\text { (M: } 14.1 \text { years) }\end{array}$ & $\begin{array}{l}\text { Nationally } \\
\text { representative } \\
\text { survey }\end{array}$ & $\begin{array}{l}\text { Video Game Addiction Test } \\
\text { (VAT) }\end{array}$ & Latent class analysis & $1.3 \%$ \\
\hline $\begin{array}{l}\text { Park et al. } \\
(2017)\end{array}$ & South Korea & 7,650 adults & $\begin{array}{l}\text { Nationally } \\
\text { representative } \\
\text { survey }\end{array}$ & DSM-5 criteria for IGD & $\begin{array}{l}\text { Endorsing at least } 5 \\
\text { criteria }\end{array}$ & $4 \%$ \\
\hline $\begin{array}{l}\text { Rosenkranz } \\
\text { et al. (2017) }\end{array}$ & Germany & $\begin{array}{l}\text { 5,667 adolescents aged be- } \\
\text { tween } 12 \text { to } 19 \text { years }(\mathrm{M}: \\
15.44, \mathrm{SD}: 1.73)\end{array}$ & $\begin{array}{l}\text { Nationally } \\
\text { representative } \\
\text { survey }\end{array}$ & AICA-S & Score above 13.5 & $3.6 \%$ \\
\hline $\begin{array}{l}\text { Wartberg et al. } \\
\text { (2017) }\end{array}$ & Germany & $\begin{array}{l}1,531 \text { adolescents and } \\
\text { young adults (M: } 18.86 \\
\text { years, SD: } 4.06)\end{array}$ & $\begin{array}{l}\text { Nationally repre- } \\
\text { sentative survey }\end{array}$ & IGDS & $\begin{array}{l}\text { Endorsing at least } 5 \\
\text { criteria }\end{array}$ & $5.7 \%$ \\
\hline
\end{tabular}


psychosocial well-being and loneliness (Lemmens, Valkenburg, \& Peter, 2011), poorer social skills (Griffiths, 2010; Zamani, Kheradmand, Cheshmi, Abedi, \& Hedayati, 2010), decreased academic achievement (Chiu, Lee, \& Huang, 2004; Jeong \& Kim, 2011; Rehbein et al., 2010; Skoric, Teo, \& Neo, 2009), increased inattention (Batthyány et al., 2009; Chan \& Rabinowitz, 2006), aggressive/ oppositional behavior and hostility (Chan \& Rabinowitz, 2006; Chiu et al., 2004), maladaptive coping (Batthyány et al., 2009; Hussain \& Griffiths, 2009a, 2009b), decreased verbal memory performance (Dworak et al., 2007), maladaptive cognitions (Peng \& Liu, 2010), and suicidal ideation (Rehbein et al., 2010).

In addition to the reported negative psychosocial consequences, there are also many reported health and medical consequences that may result from excessive video game playing. These include epileptic seizures (Chuang, 2006; Graf, Chatrian, Glass, \& Knauss, 1994; Harding \& Jeavons, 1994; Maeda et al., 1990; Millett, Fish, \& Thompson, 1997; Quirk et al., 1995); auditory hallucinations (Ortiz de Gortari \& Griffiths, 2014a; Spence, 1993); visual hallucinations (Ortiz de Gortari \& Griffiths, 2014b); enuresis (Schink, 1991); encopresis (Corkery, 1990); obesity (Deheger, Rolland-Cachera, \& Fontvielle, 1997; Johnson \& Hackett, 1997; Shimai, Yamada, Masuda, \& Tada, 1993; Vandewater, Shim, \& Caplovitz, 2004); wrist pain (McCowan, 1981); neck pain (Miller, 1991); elbow pain (Miller, 1991); tenosynovitis_also called "nintendinitis" (Brasington, 1990; Casanova \& Casanova, 1991; Reinstein, 1983; Siegal, 1991); blisters, calluses, sore tendons, and numbness of fingers (Loftus \& Loftus, 1983); hand-arm vibration syndrome (Cleary, McKendrick, \& Sills, 2002); sleep abnormalities (Allison et al., 2006; Dworak et al., 2007); psychosomatic challenges (Batthyány et al., 2009); and repetitive strain injuries (Mirman \& Bonian, 1992).

Taken together, this relatively long list of potential psychosocial and medical negative consequences indicates that excessive gaming is an issue irrespective of whether it is an addiction. It also suggests that more extensive recognition is needed of the wide range of potential negative and life-limiting consequences of excessive video play.

\section{Factors Associated with Problematic Video Game Use and Video Game Addiction}

A number of studies have examined the role of different personality factors, comorbidity factors, and biological factors and their association with gaming addiction. In relation to personality traits, gaming addiction has been shown to have association with neuroticism (Mehroof \& Griffiths, 2010; Peters \& Malesky, 2008), aggression and hostility (Caplan, Williams, \& Yee, 2009; Chiu et al., 2004; Kim, Namkoong, Ku, \& Kim, 2008; Mehroof \& Griffiths, 2010), avoidant and schizoid interpersonal tendencies (Allison et al., 2006), loneliness and introversion (Caplan et al., 2009), social inhibition (Porter, Starcevic, Berle, \& Fenech, 2010), boredom inclination (Chiu et al., 2004), sensationseeking (Chiu et al., 2004; Mehroof \& Griffiths, 2010), diminished agreeableness (Peters \& Malesky, 2008), diminished self-control and narcissistic personality traits (Kim et al., 2008), low selfesteem (Ko, Yen, Chen, Chen, \& Yen, 2005), state and trait anxiety (Mehroof \& Griffiths, 2010), and low emotional intelligence (Parker et al., 2008). It is hard to assess the etiological significance of these associations with gaming addiction as they may not be unique to the disorder. Further research is therefore needed.

Research has also shown gaming addiction to be associated with a variety of comorbid disorders. This includes attention deficit hyperactivity disorder (Allison et al., 2006; Batthyány et al., 2009; Chan \& Rabinowitz, 2006; Han et al., 2009) and symptoms of generalized anxiety disorder, panic disorder, depression, social phobia (Allison et al., 2006), school phobia (Batthyány et al., 2009), and various psychosomatic symptoms (Batthyány et al., 2009).

Through use of fMRI, biological research has shown that gaming addicts show similar neural processes and increased activity in brain areas associated with substance-related addictions and other behavioral addictions, such as pathological gambling (significant activation in the left occipital lobe, parahippocampal gyrus, dorsolateral prefrontal cortex, nucleus accumbens, right orbitofrontal cortex, bilateral anterior cingulate, medial frontal cortex, and the caudate nucleus (Han et al., 2010; Hoeft et al., 2008; Ko et al., 2009). It has also been reported that gaming addicts (like substance addicts) have a higher prevalence of two specific polymorphisms of the dopaminergic system (i.e., Taq1A1 allele of the dopamine $\mathrm{D}_{2}$ receptor and the Val158Met in the catecholamine-o-methyltransferase receptor) (Han et al., 2007).

\section{Internet Gaming Disorder and the DSM-5}

Prior to the publication of the DSM-5 (American Psychiatric Association, 2013), there had been some 
debate as to whether "Internet addiction" should be introduced into the text as a separate disorder (Block, 2008; Petry \& O’Brien, 2013). Alongside this, there was debate as to whether those researching the online addiction field should be researching generalized Internet use and/or the potentially addictive activities that can be engaged on the Internet (e.g., gambling, video gaming, sex, shopping, etc.) (Griffiths, 2000; Griffiths, King, \& Demetrovics, 2014). Following these debates, the Substance Use Disorder Work Group (SUDWG) recommended that the DSM-5 include a subtype of problematic Internet use (i.e., Internet gaming disorder [IGD]) in Section 3 ("Emerging Measures and Models") as an area that needed future research before being included in future editions of the DSM (Petry \& O'Brien, 2013). According to Petry and O'Brien (2013), IGD will not be included as a separate mental disorder until the (1) defining features of IGD have been identified, (2) reliability and validity of specific IGD criteria have been obtained cross-culturally, (3) prevalence rates have been determined in representative epidemiological samples across the world, and (4) etiology and associated biological features have been evaluated.

One of the key reasons that IGD was not included in the main text of the DSM-5 was that the SUDWG concluded that no standard diagnostic criteria were used to assess gaming addiction across these many studies (Griffiths et al., 2014). A review of instruments assessing problematic, pathological, and/or addictive gaming by King and colleagues (2013) reported that 18 different screening instruments had been developed and that these had been used in 63 quantitative studies comprising 58,415 participants. This comprehensive review identified both strengths and weaknesses of these instruments. The main strengths of the instrumentation included (1) the brevity and ease of scoring; (2) excellent psychometric properties, such as convergent validity and internal consistency; and (3) robust data that will aid the development of standardized norms for adolescent populations. However, the main weaknesses identified in the instrumentation included (1) core addiction indicators being inconsistent across studies, (2) a general lack of any temporal dimension, (3) inconsistent cutoff scores relating to clinical status, (4) poor and/or inadequate interrater reliability and predictive validity; and (5) inconsistency and/or dimensionality. It has also been noted by a number of authors that the criteria for IGD assessment tools are theoretically based on a variety of different potentially problematic activities including substance use disorders, pathological gambling, and/or other behavioral addiction criteria (King et al., 2013; Petry \& O’Brien, 2013). There are also issues surrounding the settings in which diagnostic screens are used, as those used in clinical practice settings may require a different emphasis than those used in epidemiological, experimental, and neurobiological research settings (King et al., 2013; Koronczai et al., 2011).

A recent review by Pápay and colleagues (2014) argued that some researchers consider video games as the starting point for examining the characteristics of IGD, while others consider the Internet as the main platform that unites different addictive Internet activities, including online games. Recent studies (Demetrovics et al., 2012; Kim \& Kim, 2010) have made an effort to integrate both approaches. Consequently, IGD can either be viewed as a specific type of video game addiction, as a variant of Internet addiction, or as an independent diagnosis (Griffiths et al., 2014).

Griffiths (2005) has argued that although all addictions have particular and idiosyncratic characteristics, they share more commonalities than differences (i.e., salience, mood modification, tolerance, withdrawal symptoms, conflict, and relapse) and likely reflect a common etiology of addictive behavior. Consequently, online game addiction is viewed as a specific type of video game addiction. Similarly, Porter and colleagues (2010) do not differentiate between problematic video game use and problematic online game use. They conceptualized problematic video game use as excessive use of one or more video games resulting in a preoccupation with and a loss of control over playing video games and their various negative psychosocial and/or physical consequences. However, unlike Griffiths (2005), their criteria for problematic video game use does not include other features usually associated with dependence or addiction (e.g., tolerance, physical symptoms of withdrawal) as they say there is no clear evidence that problematic gaming is associated with such phenomena. Researchers such as Young (1998b) view online gaming addiction as a subtype of Internet addiction, as they believe the Internet itself provides situation-specific characteristics that facilitate gaming becoming problematic and/or addictive.

Kim and Kim's (2010) Problematic Online Game Use (POGU) model takes a more integrative approach and claims that neither of the approaches 
just outlined adequately capture the unique features of online games such as MMORPGs. They argue that the Internet is just one channel where people may access the content they want (e.g., gambling, shopping, sex, etc.) and that such users may become addicted to the particular content rather than to the channel itself. This is analogous to the argument by Griffiths (2000) that there is a fundamental difference between addiction to the Internet and addictions on the Internet. MMORPGs also differ from single-player video games as there are social and/or role-playing dimensions that allow interaction with other gamers.

The POGU model resulted in five underlying dimensions of addictive gameplay (i.e., euphoria, health problems, conflict, failure of self-control, and preference of virtual relationships). Demetrovics and colleagues (2012) also support the integrative approach and stress the need to include all types of online games in addiction models in order to make comparisons between genres and gamer populations possible (such as those who play online real-time strategy (RTS) games and online first-person shooter (FPS) games in addition to the widely researched MMORPG players). Their model comprises six dimensions (i.e., preoccupation, overuse, immersion, social isolation, interpersonal conflicts, and withdrawal).

Irrespective of approach or model, the components and dimensions that comprise online gaming addiction just outlined are very similar to the IGD criteria in Section 3 of the DSM-5. For instance, Griffiths's (2005) six addiction components directly map onto the nine proposed criteria for IGD (of which five or more need to be endorsed and result in clinically significant impairment). More specifically: (1) preoccupation with Internet games (salience); (2) withdrawal symptoms when Internet gaming is taken away (withdrawal); (3) the need to spend increasing amounts of time engaged in Internet gaming (tolerance), (4) unsuccessful attempts to control participation in Internet gaming (relapse/loss of control); (5) loss of interest in hobbies and entertainment as a result of, and with the exception of, Internet gaming (conflict); (6) continued excessive use of Internet games despite knowledge of psychosocial problems (conflict); (7) deception of family members, therapists, or others regarding the amount of Internet gaming (conflict); (8) use of the Internet gaming to escape or relieve a negative mood (mood modification); and (9) loss of a significant relationship, job, or educational or career opportunity because of participation in Internet games (conflict).

\section{Treatment of Gaming Addiction}

Clinical interventions and treatment for problematic and/or addictive gaming vary considerably in the literature, with most of the very few published studies employing some type of CBT, pharmacotherapy, and/or self-devised psychological interventions (Griffiths \& Meredith, 2009; Han et al., 2009, 2010; King, Delfabbro, \& Griffiths, 2010, 2012). Han et al. (2010) presented some successful case studies regarding pharmacotherapeutic treatment. After a 6-week (Han et al., 2010) and a 12-week (Han \& Renshaw, 2012) period of bupropion sustained-release treatment, problematic gamers showed significant improvement, evident in both decreased problem behavior and decreased depression scores. The researchers' pharmacological choice had been driven by the similarities in neurological activity of different behavioral addictions (Han et al., 2010; Ko et al., 2009; Kuss \& Griffiths, 2012).

Currently, the evidence base on the treatment of problematic and/or addictive gaming is limited. Furthermore, the lack of consistent approaches to treating problematic video game playing and video game addiction makes it difficult to produce any definitive conclusions as to the efficacy of treatment, although at this stage CBT (as with the treatment efficacy of other addictions) appears to show good preliminary support (King et al., 2012). There remains a need for controlled, comparative studies of psychological and pharmacological treatments, administered individually and in combination with each other, to determine the optimal treatment approach.

The lack of comparative treatment studies might suggest that there is a general lack of demand for psychological services for problematic video game play and/or video game addiction (King et al., 2010). However, this may not necessarily be the case. For instance, Woog (2004) surveyed a random sample of 5,000 US mental health professionals. Although only 229 participants completed the questionnaire, two-thirds had treated someone with excessive computer use problems in the year prior to the survey. Woog also reported that problematic gaming was most common among 11- to 17-year-old clients. However, this may not be truly representative as this age group may be more likely to present in therapy. Anecdotal evidence suggests that 11- to 17-year-old 
clients are typically forced by concerned parents to attend treatment. Adult gaming addicts may not seek treatment or may seek treatment at a later stage for other psychological problems (e.g., depression) that develop after experiencing the severe negative consequences of gaming.

In South East Asia there appears to be significant demand for treatment for online-related problems, including gaming addiction. The South Korean government has reportedly established a network of more than 140 counseling centers for treatment of online addiction (Kim, 2008). In Western countries, gaming addiction clinics have also started to emerge in places such as Holland and the United Kingdom (Griffiths \& Meredith, 2009; King, Delfabbro, Griffiths, \& Gradisar, 2011). There are also treatment groups that are modeled on 12-step self-help treatment (e.g., Online Gamers Anonymous) (Griffiths \& Meredith, 2009). However, little detail is known about the treatment protocols or their efficacy.

\section{Conclusion}

Based on the published empirical studies, and particularly those published over the past decade, it appears that, in extreme cases, excessive gaming can have potentially damaging effects on individuals who appear to display compulsive and/or addictive behavior similar to other more traditional addictions. However, the field has been hindered by the use of inconsistent and nonstandardized criteria to assess and identify problematic and/or addictive video game use. Furthermore, most studies' recruitment methods have serious sampling biases, with an overreliance on self-selected samples.

Despite these shortcomings, there are several noticeable trends that can be drawn from this review of problematic video game play and gaming addiction.

- There has been a significant increase in empirical research decade by decade since the early 1980s.

- There has been a noticeable (and arguably strategic) shift in researching the mode of video game play. In the 1980 s, research mainly concerned "pay-to-play" arcade video games. In the 1990s, research mainly concerned stand-alone (offline) video games played at home on consoles, PCs or handheld devices. In the 2000s, research mainly concerned online massively multiplayer video games.

- There has been a noticeable shift in how data are collected. Up until the early 2000s, data about video game behavior were typically collected face to face, whereas contemporary studies collect data online, strategically targeting online forums where gamers are known to (virtually) congregate. These samples are typically self-selecting and (by default) unrepresentative of the general population. Therefore, generalization is almost always one of the methodological shortcomings of this data collection approach.

- Survey study sample sizes have generally increased. In the 1980s and 1990s, sample sizes were typically in the low hundreds. In the 2000s, sample sizes in the thousands-even if unrepresentative-are not uncommon.

- There has been a diversification in the way data are collected, including experiments, physiological investigations, secondary analysis of existing data (such as data collected from online forums), and behavioral tracking studies.

- There has been increased research on adult (i.e., non-child and non-adolescent) samples, reflecting the fact that the demographics of gaming have changed.

- There has been increasing sophistication in relation to issues concerning assessment and measurement of problematic video game play and video game addiction. In the past few years, instruments have been developed that have more robust psychometric properties in terms of reliability and validity. However, there are still some concerns as many of the most widely used screening instruments were adapted from adult screens and much of the video game literature has examined children and adolescents. King et al. (2012) assert that to enable future advances in the development and testing of interventions for video game-related problems, there must be some consensus among clinicians and researchers as to the precise classification of these problems.

The fact that IGD was included in Section 3 of the DSM-5 appears to have been well received by researchers and clinicians in the gaming addiction field (and by those individuals who have sought treatment for such disorders and had their experiences psychiatrically validated). However, for IGD to be included in the section on "Substance-Related and Addictive Disorders" along with "Gambling Disorder," the gaming addiction field must unite and start using the same assessment measures so that comparisons can be made across different demographic groups and different cultures. For epidemiological purposes, Koronczai and colleagues 
(2011) assert that the most appropriate measures in assessing problematic online use (including Internet gaming) should meet six requirements. Such an instrument should have (1) brevity (to make surveys as short as possible and help overcome question fatigue), (2) comprehensiveness (to examine all core aspects of problematic gaming as possible), (3) reliability and validity across age groups (e.g., adolescents vs. adults), (4) reliability and validity across data collection methods (e.g., online, face-to-face interview, paper-and-pencil), (5) cross-cultural reliability and validity, and (6) clinical validation. It was also noted that an ideal assessment instrument should serve as the basis for defining adequate cutoff scores in terms of both specificity and sensitivity.

Clearly, there exist a number of gaps in our current understanding of problematic video game play and gaming addiction. King, Delfabbro, and Griffiths (2013) note a need for epidemiological research to determine the incidence and prevalence of clinically significant problems associated with video game play in the broader population. There are too few clinical studies that describe the unique features and symptoms of problematic video game play and/ or video game addiction. Most of the studies tend to examine problematic video play from the perspective of the individual. However, there is a small body of research suggesting that the characteristics of the video games themselves may have a role in the acquisition, development, and maintenance of video game addiction. These studies have investigated the role of structural characteristics of video games in maintaining problem playing behavior (King et al., 2011; Westwood \& Griffiths, 2010; Wood, Griffiths, Chappell, \& Davies, 2004), but there is little empirical research that examines why some individuals may be protected from developing excessive playing habits or whether some individuals simply mature out of their problem playing behavior.

Another growing concern is the recent explosion of online and mobile gaming although, as yet, little research has been done. There are also strong links between online gaming, gambling, non-gambling fantasy games, role-playing games, board games, and card games. These may be an additional cause for concern as youth migrate from free gaming sites to online gambling sites. It should also be noted that video game playing does not occur in a vacuum, but is one behavior engaged in alongside many others. To date, very few studies have been used to examine links between video games and other risk behaviors (e.g., gambling, drug and alcohol use, seatbelt use, poor school performance, conduct problems, truancy, delinquency, violence and sexual activity).

\section{Acknowledgments}

Some of the material in this chapter was previously published in the following previous works:

Griffiths, M. D., Kuss, D. J., \& King, D. L. (2012). Video game addiction: Past, present and future. Current Psychiatry Reviews, 8(4), 308-18. doi:10.2174/157340012803520414

Griffiths, M. D. (2015). Online games, addiction and overuse of. In The International Encyclopedia of Digital Communication and Society. Chichester: John Wiley \& Sons, Inc.

King, D. L., Delfabbro, P. H., \& Griffiths, M. D. (2013). Chapter 82 - Video Game Addiction. In P. M. Miller (Ed.), Principles of Addiction (pp. 819-25). San Diego: Academic Press.

\section{References}

Allison, S. E., von Wahlde, L., Shockley, T., \& Gabbard, G. O. (2006). The development of the self in the era of the Internet and role-playing fantasy games. American Journal of Psychiatry, 163, 381-85.

American Psychiatric Association. (1987). Diagnostic and Statistical Manual for Mental Disorders (3rd ed.). Washington, DC: Author.

American Psychiatric Association. (1994). Diagnostic and Statistical Manual for Mental Disorders (4th ed.). Washington, DC: Author.

American Psychiatric Association. (2013). Diagnostic and Statistical Manual of Mental Disorders-Text Revision (5th ed.). Washington, DC: Author.

Barnett, J., \& Coulson, M. (2010). Virtually real: A psychological perspective on massively multiplayer online games. Review of General Psychology, 4, 167-79.

Batthyány, D., Müller, K. W., Benker, F., \& Wölfling, K. (2009). Computer game playing: Clinical characteristics of dependence and abuse among adolescents. Wiener Klinsche Wochenschrift, 121(15-16), 502-09.

Blocher, J. M. (2015). Gaming. In R. Papa (Ed.), Media Rich Instruction (pp. 219-34). Champaign, IL: Springer International Publishing.

Block, J. J. (2008). Issues for DSM-V: Internet addiction [Editorial]. American Journal of Psychiatry, 165, 306.

Brasington, R. (1990). Nintendinitis. New England Journal of Medicine, 322, 1473-74.

Brown, R. I. F., \& Robertson, S. (1993). Home computer and video game addictions in relation to adolescent gambling: Conceptual and developmental aspects. In W. R. Eadington \& J. A. Cornelius (Eds.), Gambling behavior and problem gambling (pp. 451-71). Reno: University of Nevada Press.

Caplan, S. E., Williams, D., \& Yee, N. (2009). Problematic internet use and psychosocial well-being among MMO players. Computers in Human Behavior, 25, 1312-19.

Casanova, J., \& Casanova, J. (1991). Nintendinitis. Journal of Hand Surgery, 16, 181.

Chan, P. A., \& Rabinowitz, T. (2006). A cross-sectional analysis of video games and attention deficit hyperactivity disorder symptoms in adolescents. Annals of General Psychiatry, 5(1), 16-26.

Charlton, J. P. (2002). A factor-analytic investigation of computer 'addiction' and engagement. British Journal of Psychology, 93, 329-44. 
Chiu, S. I., Lee, J. Z., \& Huang, D. H. (2004). Video game addiction in children and teenagers in Taiwan. CyberPsychology and Behavior, 7, 571-81.

Chuang, Y. C. (2006). Massively multiplayer online role-playing game-induced seizures: A neglected health problem in Internet addiction. CyberPsychology and Behavior, 9, 451-56.

Cleary, A. G., Mckendrick, H., \& Sills, J. A. (2002). Handarm vibration syndrome may be associated with prolonged use of vibrating computer games. British Medical Journal, 324,301

Cole, H. \& Griffiths, M. D. (2007). Social interactions in Massively Multiplayer Online Role-Playing gamers. CyberPsychology and Behavior, 10, 575-583.

Corkery, J. C. (1990). Nintendo power. American Journal of Diseases in Children, 144, 959.

Cultrara, A., \& Har-El, G. (2002). Hyperactivity-induced suprahyoid muscular hypertrophy secondary to excessive video game play: A case report. Journal of Oral and Maxillofacial Surgery, 60, 326-27.

Deheger, M., Rolland-Cachera, M. F., \& Fontvielle, A. M. (1997). Physical activity and body composition in 10-yearold French children: Linkages with nutritional intake? International Journal of Obesity, 21, 372-79.

Demetrovics, Z., Urbán, R., Nagygyörgy, K., Farkas, J., Griffiths, M. D., Pápay, O., . . Oláh, A. (2012). The development of the Problematic Online Gaming Questionnaire (POGQ). Plos One, 7(5), e36417.

Dreier, M., Wölfling, K., \& Müller, K. W. (2013). Psychological research and a sociological perspective on problematic and addictive computer game use in adolescents. In A. Tsitsika, M. Janikian, D. E. Greydanus, H. A. Omar, \& J. Merrick (Eds.), Internet addiction: A public health concern in adolescence (pp. 87-110). New York: Nova Science Publishers.

Dworak, M., Schierl, T., Bruns, T., \& Struder, H. K. (2007). Impact of singular excessive computer game and television exposure on sleep patterns and memory performance of school-aged children. Pediatrics, 120, 978-85.

Entertainment Software Association. (2014). Essential facts about the computer and video game industry. Washington, DC: Entertainment Software Association. www.theesa.com/facts/ pdfs/esa_ef_2013.pdf

Fisher, S. E. (1994). Identifying video game addiction in children and adolescents. Addictive Behaviors, 19, 545-53.

Gentile, D. A. (2009). Pathological video-game use among youth ages 8 to 18: A national study. Psychological Science, 20(5), 594-602.

Gentile, D. A., Choo, H., Liau, A., Sim, T., Li, D. D., Fung, D., \& Khoo, A. (2011). Pathological video game use among youths: A two-year longitudinal study. Pediatrics, 127(2), 319-29.

Graf, W. D., Chatrian, G. E., Glass, S. T., \& Knauss, T. A. (1994). Video-game related seizures: A report on 10 patients and a review of the literature. Pediatrics, 3, 551-56.

Griffiths, M. D. (1997). Computer game playing in early adolescence. Youth and Society, 29, 223-37.

Griffiths, M. D. (2000). Internet addiction-Time to be taken seriously? Addiction Research, 8, 413-18.

Griffiths, M. D. (2005). A 'components' model of addiction within a biopsychosocial framework. Journal of Substance Use, 10, 191-97.

Griffiths, M. D. (2010). Computer game playing and social skills: A pilot study. Aloma: Revista de Psicologia, Ciències de l'Educació i de l'Esport, 27, 301-10.
Griffiths, M. D., Davies, M. N. O., \& Chappell, D. (2004). Demographic factors and playing variables in online computer gaming. CyberPsychology and Behavior, 7, 479-87.

Griffiths, M. D., \& Hunt, N. (1995). Computer game playing in adolescence: Prevalence and demographic indicators. Journal of Community and Applied Social Psychology, 5, 189-93.

Griffiths, M. D., \& Hunt, N. (1998). Dependence on computer games by adolescents. Psychological Reports, 82, 475-80.

Griffiths, M. D., King, D. L., \& Demetrovics, Z. (2014). DSM-5 Internet gaming disorder needs a unified approach to assessment. Neuropsychiatry, 4(1), 1-4.

Griffiths, M. D., \& Meredith, A. (2009). Videogame addiction and treatment. Journal of Contemporary Psychotherapy, 39(4), 47-53.

Grüsser, S. M., Thalemann, R., \& Griffiths, M. D. (2007). Excessive computer game playing: Evidence for addiction and aggression? CyberPsychology and Behavior, 10, 290-92.

Han, D. H., Hwang, J. W., \& Renshaw, P. F. (2010). Bupropion sustained release treatment decreases craving for video games and cue-induced brain activity in patients with Internet video game addiction. Experimental and Clinical Psychopharmacology, 18, 297-304.

Han, D. H., Lee, Y. S., Na, C., Ahn, J. Y., Chung, U. S., Daniels, M. A., ... Renshaw, P. F. (2009). The effect of methylphenidate on Internet video game play in children with attention-deficit/ hyperactivity disorder. Comprehensive Psychiatry, 50, 251-56.

Han, D. H., Lee, Y. S., Yang, K. C., Kim, E. Y., Lyoo, I. K., \& Renshaw, P. F. (2007). Dopamine genes and reward dependence in adolescents with excessive internet video game play. Journal of Addiction Medicine, 1, 133-38.

Han, D. H., \& Renshaw, P. F. (2012). Bupropion in the treatment of problematic online game play in patients with major depressive disorder. Journal of Psychopharmacology, 26, 689-696.

Harding, G. F. A., \& Jeavons, P. M. (1994). Photosensitive epilepsy. London: Mac Keith Press.

Hoeft, F., Watson, C. L., Kesler, S. R., Bettinger, K. E., \& Reiss, A. L. (2008). Gender differences in the mesocorticolimbic system during computer game-play. Journal of Psychiatric Research, 42, 253-58.

Hussain, Z., \& Griffiths, M. D. (2009a). The attitudes, feelings, and experiences of online gamers: A qualitative analysis. CyberPsychology and Behavior, 12, 747-53.

Hussain, Z., \& Griffiths, M. D. (2009b). Excessive use of massively-multi-player online role-playing games: A pilot study. International Journal of Mental Health and Addiction, 7, 563-71.

Jansz, J., \& Tanis, M. (2007). Appeal of playing online first person shooter games. CyberPsychology \& Behavior, 10(1), 133-136.

Jeong, E. J., \& Kim, D. W. (2011). Social activities, self-efficacy, game attitudes, and game addiction. Cyberpsychology, Behavior \& Social Networking, 14, 213-21.

Johnson, B., \& Hackett, A. F. (1997). Eating habits of 11-14-yearold schoolchildren living in less affluent areas of Liverpool, UK. Journal of Human Nutrition and Dietetics, 10, 135-44.

Johnston, B., Boyle, L., MacArthur, E., \& Manion, B. F. (2013). The role of technology and digital gaming in nurse education. Nursing Standard, 27(28), 35-38.

Keepers, G. A. (1990). Pathological preoccupation with video games. Journal of the American Academy of Child and Adolescent Psychiatry, 29, 49-50.

Kim, E. J., Namkoong, K., Ku, T., \& Kim, S. J. (2008). The relationship between online game addiction and aggression, self-control, and narcissistic personality traits. European Psychiatry, 23, 212-18. 
Kim, J. (2008). The effect of a R/T group counselling program on the Internet addiction level and self-esteem of Internet addiction university students. International Journal of Reality Therapy, 17, 4-12.

Kim, M. G., \& Kim, J. (2010). Cross-validation of reliability, convergent and discriminant validity for the problematic online game use scale. Computers in Human Behavior, 26, 389-98.

King, D. L., \& Delfabbro, P. (2009). Understanding and assisting excessive players of video games: A community psychology perspective. The Australian Community Psychologist, 21(1), 62-74.

King, D. L., Delfabbro, P. H., \& Griffiths, M. D. (2010). Cognitive behavioural therapy for problematic video game players: Conceptual considerations and practice issues. Journal of CyberTherapy and Rehabilitation, 3, 261-73.

King, D. L., Delfabbro, P. H., \& Griffiths, M. D. (2011). The role of structural characteristics in problematic video game play: An empirical study. International Journal of Mental Health and Addiction, 9, 320-33.

King, D. L., Delfabbro, P. H., \& Griffiths, M. D. (2012). Clinical interventions for technology-based problems: Excessive Internet and video game use. Journal of Cognitive Psychotherapy, 26, 43-56.

King, D. L., Delfabbro, P. H., \& Griffiths, M. D. (2013). Video game addiction. In P. Miller (Ed.), Principles of addiction: Comprehensive addictive behaviors and disorders. (Vol. 1, pp. 819-25). San Diego: Academic Press.

King, D. L., Delfabbro, P. H., Griffiths, M. D., \& Gradisar, M. (2011). Assessing clinical trials of Internet addiction treatment: A systematic review and CONSORT evaluation. Clinical Psychology Review, 31, 1110-16.

King, D. L., Haagsma, M. C., Delfabbro, P. H., Gradisar, M. S., \& Griffiths, M. D. (2013). Toward a consensus definition of pathological video-gaming: A systematic review of psychometric assessment tools. Clinical Psychology Review, 33, 331-42.

Ko, C. H., Liu, G. C., Hsiao, S. M., Yen, J. Y., Yang, M. J., Lin, W. C., et al. (2009). Brain activities associated with gaming urge of online gaming addiction. Journal of Psychiatric Research, 43, 739-47.

Ko, C. H., Yen, J. Y., Chen, C. C., Chen, S. H., \& Yen, C. F. (2005). Gender differences and related factors affecting online gaming addiction among Taiwanese adolescents. Journal of Nervous and Mental Disease, 193, 273-77.

Koronczai, B., Urban, R., Kokonyei, G., Paksi, B., Papp, K., Kun, B., . . . Demetrovics, Z. (2011). Confirmation of the three-factor model of problematic internet use on off-line adolescent and adult samples. Cyberpsychology, Behavior and Social Networking, 14, 657-64.

Kuczmierczyk, A. R., Walley, P. B., \& Calhoun, K. S. (1987). Relaxation training, in vivo exposure and response-prevention in the treatment of compulsive video-game playing. Scandinavian Journal of Behaviour Therapy, 16, 185-90.

Kuss, D. J., \& Griffiths, M. D. (2012). Online gaming addiction: A systematic review. International Journal of Mental Health and Addiction, 10, 278-96.

Lemmens, J. S., Valkenburg, P. M., \& Peter, J. (2011). Psychosocial causes and consequences of pathological gaming. Computers in Human Behavior, 27, 144-52.

Liu, M., \& Peng, W. (2009). Cognitive and psychological predictors of the negative outcomes associated with playing MMOGs (massively multiplayer online games). Computers in Human Behavior, 25, 1306-11.
Loftus, G. A., \& Loftus, E. F. (1983). Mind at play: The psychology of video games. New York: Basic Books.

Maeda, Y., Kurokawa, T., Sakamoto, K., Kitamoto, I., Kohji, U., \& Tashima, S. (1990). Electroclinical study of video-game epilepsy. Developmental Medicine and Child Neurology, 32, 493-500.

McCowan, T. C. (1981). Space Invaders wrist. New England Journal of Medicine, 304, 1368.

Mehroof, M., \& Griffiths, M. D. (2010). Online gaming addiction: The role of sensation seeking, self-control, neuroticism, aggression, state anxiety, and trait anxiety. CyberPsychology and Behavior, 13, 313-16.

Miller, D. L. G. (1991). Nintendo neck. Canadian Medical Association Journal, 145, 1202.

Millett, C. J., Fish, D. R., \& Thompson, P. J. (1997). A survey of epilepsy-patient perceptions of video-game material/ electronic screens and other factors as seizure precipitants. Seizure, 6, 457-59.

Mirman, M. J., \& Bonian, V. G. (1992). "Mouse elbow": A new repetitive stress injury. Journal of the American Osteopath Association, 92, 701.

Nagygyörgy, K., Urbán, R., Farkas, J., Griffiths, M. D., Zilahy, D., Kökönyei, G., . . . \& Harmath, E. (2013). Typology and sociodemographic characteristics of massively multiplayer online game players. International Journal of HumanComputer Interaction, 29(3), 192-200.

Nilles, J. M. (1982). Exploring the world of the personal computer. Englewood Cliffs, NJ: Prentice Hall.

Ortiz de Gortari, A. B., \& Griffiths, M. D. (2014a). Auditory experiences in Game Transfer Phenomena: An empirical selfreport study. International Journal of Cyber Behavior, Psychology and Learning, 4(1), 59-75.

Ortiz de Gortari, A. B., \& Griffiths, M. D. (2014b). Altered visual perception in Game Transfer Phenomena: An empirical self-report study. International Journal of HumanComputer Interaction, 30, 95-105.

Pápay, O., Nagygyörgy, K., Griffiths, M. D., \& Demetrovics, Z. (2014). Problematic online gaming. In K. Rosenberg \& L. Feder (Eds.), Behavioral addictions: Criteria, evidence and treatment (pp. 61-95). New York: Elsevier.

Pápay, O., Urbán, R., Griffiths, M. D., Nagygyörgy, K., Farkas, J., Elekes, Z., . . . Demetrovics, Z. (2013). Psychometric properties of the Problematic Online Gaming Questionnaire Short-Form (POGQ-SF) and prevalence of problematic online gaming in a national sample of adolescents. Cyberpsychology, Behavior and Social Networking, 16, 340-48.

Parker, J. D. A., Taylor, R. N., Eastabrook, J. M., Schell, S. L., \& Wood, L. M. (2008). Problem gambling in adolescence: Relationships with internet misuse, gaming abuse and emotional intelligence. Personality and Individual Differences, 45(2), 174-80.

Parsons, K. (1995, April). Educational places or terminal cases: Young people and the attraction of computer games. Paper presented at the British Sociological Association Annual Conference, University of Leicester.

Peng, W., \& Liu, M. (2010). Online gaming dependency: A preliminary study in China. Cyberpsychology, Behavior and Social Networking, 13, 329-33.

Peters, C. S., \& Malesky, L. A. (2008). Problematic usage among highly-engaged players of massively multiplayer online roleplaying games. CyberPsychology and Behavior, 11, 480-83.

Petry, N. M., \& O’Brien, C. P. (2013). Internet gaming disorder and the DSM-5. Addiction, 108, 1186-87. 
Phillips, C. A., Rolls, S., Rouse, A., \& Griffiths, M. D. (1995). Home video game playing in schoolchildren: A study of incidence and pattern of play. Journal of Adolescence, 18, 687-91.

Pontes, H., \& Griffiths, M. D. (2014). The assessment of internet gaming disorder in clinical research. Clinical Research and Regulatory Affairs, 31(2-4), 35-48.

Porter, G., Starcevic, V., Berle, D., \& Fenech, P. (2010). Recognizing problem video game use. The Australian and New Zealand Journal of Psychiatry, 44(2), 120-28.

Przybylski, A. K. (2014). Electronic gaming and psychosocial adjustment. Pediatrics, 134(3), e716-e722.

Quirk, J. A., Fish, D. R., Smith, S. J. M., Sander, J. W., Shorvon, S. D., \& Allen, P. J. (1995). First seizures associated with playing electronic screen games: A community based study in Great Britain. Annals of Neurology, 37, 110-24.

Rehbein, F., Kleimann, M., \& Mossle, T. (2010). Prevalence and risk factors of video game dependency in adolescence: Results of a German nationwide survey. CyberPsychology, Behavior and Social Networking, 13, 269-77.

Reinstein, L. (1983). De Quervain's stenosing tenosynovitis in a video games player. Archives of Physical and Medical Rehabilitation, 64, 434-35.

Schink, J. C. (1991). Nintendo enuresis. American Journal of Diseases in Children, 145, 1094.

Shimai, S., Yamada, F., Masuda, K., \& Tada, M. (1993). TV game play and obesity in Japanese school children. Perceptual and Motor Skills, 76, 1121-22.

Shotton, M. (1989). Computer addiction? A study of computer dependency. London: Taylor and Francis.

Siegal, I. M. (1991). Nintendonitis. Orthopedics, 14, 745.

Skoric, M. M., Teo, L. L. C., \& Neo, R. L. (2009). Children and video games: Addiction, engagement, and scholastic achievement. CyberPsychology and Behavior, 12, 567-72.

Soper, W. B., \& Miller, M. J. (1983). Junk time junkies: An emerging addiction among students. School Counsellor, 31, $40-43$.

Spence, S. A. (1993). Nintendo hallucinations: A new phenomenological entity. Irish Journal of Psychological Medicine, 10, 98-99.

Thalemann, R., Wölfling, K., \& Grüsser, S. M. (2007). Specific cue reactivity on computer game-related cues in excessive gamers. Behavioral Neuroscience, 12, 614-18.

Thomas, N. J., \& Martin, F. H. (2010). Video-arcade game, computer game and Internet activities of Australian students:
Participation habits and prevalence of addiction. Australian Journal of Psychology, 62, 59-66.

Vandewater, E. A., Shim, M., \& Caplovitz, A. G. (2004). Linking obesity ad activity level with children's television and game use. Journal of Adolescence, 27, 71-85.

Van Rooij, A. J., Schoenmakers, T. M., Vermulst, A. A., Van den Eijnden, R. J., \& Van de Mheen, D. (2011). Online video game addiction: Identification of addicted adolescent gamers. Addiction, 106(1), 205-12.

Westwood, D., \& Griffiths, M. D. (2010). The role of structural characteristics in video game play motivation: A Q-Methodology study. Cyberpsychology, Behavior and Social Networking, 13, 581-85.

Widyanto, L., Griffiths, M. D., \& Brunsden, V. (2011). A psychometric comparison of the Internet Addiction Test, the Internet Related Problem Scale, and self-diagnosis. Cyberpsychology, Behavior, and Social Networking, 14, 141-49.

Wood, R. T. A., Griffiths, M. D., Chappell, D., \& Davies, M. N. O. (2004). The structural characteristics of video games: A psycho-structural analysis. CyberPsychology and Behavior, 7, 1-10.

Woog, K. (2004). A survey of mental health professionals' clinical exposure to problematic computer use. Unpublished study. http://www.wooglabs.com/

Yee, N. (2006a). The demographics, motivations and derived experiences of users of massively-multiuser online graphical environments. PRESENCE: Teleoperators and Virtual Environments, 15, 309-29.

Yee, N. (2006b). The psychology of MMORPGs: Emotional investment, motivations, relationship formation, and problematic usage. In R. Schroeder \& A. Axelsson (Eds.), Avatars at work and play: Collaboration and interaction in shared virtual environments (pp. 187-207). London: Springer.

Young, K. (1998a). Caught in the net. Chichester, UK: Wiley.

Young, K. S. (1998b). Internet addiction: The emergence of a new clinical disorder. Cyberpsychology and Behavior, 1, 237-44.

Yousafzai, S., Hussain, Z., \& Griffiths, M. D. (2013). Social responsibility in online videogaming: What should the videogame industry do? Addiction Research \& Theory, 22(3), 181-85.

Zamani, E., Kheradmand, A., Cheshmi, M., Abedi, A., \& Hedayati, N. (2010). Comparing the social skills of students addicted to computer games with normal students. Journal of Addiction and Health, 2, 59-69. 